\title{
Programa de ejercicios excéntricos en tendinopatías para atletas de alto rendimiento
}

\section{Eccentric exercise program in tendinopathies for high performance athletes}

\author{
Pamela Guadalupe Castro-Maldonado ${ }^{1}$ \\ 'Centro Médico Nacional 20 de Noviembre, Ciudad de México, México.
}

Cómo citar: Castro-Maldonado, P.G. 2021. Programa de ejercicios excéntricos en tendinopatías para atletas de alto rendimiento. Rev. Digit. Act. Fis. Deport. 7(1):e1674. http://doi.org/10.31910/rdafd.v7.n1.2021.1674

Artículo de acceso abierto publicado por Revista Digital: Actividad Física y Deporte, bajo una licencia Creative Commons CC BY-NC 4.0

Publicación oficial de la Universidad de Ciencias Aplicadas y Ambientales U.D.C.A, Institución de Educación Superior Acreditada de Alta Calidad por el Ministerio de Educación Nacional.

Recibido: Agosto 9 de 2020 Aceptado: Noviembre 15 de 2020 Editado por: Álvaro José Gracia Díaz

\section{RESUMEN}

Introducción: En 2017, en la Ciudad de México, se realizó una estadística sobre las principales patologías en atletas de alto rendimiento. Basado en los hallazgos encontrados, se revisan los conceptos, tratamientos y abordajes, con relación a las tendinopatías. Se describen protocolos de ejercicios excéntricos, que demostraron la eficacia del ejercicio excéntrico. La eficacia de este tipo de ejercicio, se debe a la respuesta de los tendones al entrenamiento excéntrico. La tendinopatía, nombre colectivo para tendinitis, tendinosis y paratendinitis, es el síndrome clínico, que describe las lesiones por uso excesivo del tendón. Se caracteriza por una combinación de dolor, inflamación difusa o localizada y pérdida de la función, daño térmico y respuestas compresivas adaptativas. Objetivo: Establecer la eficacia de los protocolos de ejercicios excéntricos, desde su concepción hasta su aplicación, a padecimientos de tendinopatía y enunciar la ventaja que tienen sobre otros tipos de ejercicios. Metodología: Se describen los protocolos de ejercicios excéntricos, que demostraron la eficacia del ejercicio excéntrico. La eficacia de este tipo de ejercicio, se debe a la respuesta de los tendones al entrenamiento excéntrico. Resultados: En esta revisión bibliográfica, se reportan que los programas de ejercicios excéntricos, bien diseñados, mejoran significativamente a los pacientes con tendinopatías y los resultados se mantienen a medio-largo plazo. Conclusiones: La revisión de la literatura que aquí se presenta arroja luz sobre los beneficios que tienen los ejercicios excéntricos, especialmente, en el proceso metabólico y en las propiedades mecánicas en el tendón, lo cual, específicamente, ayuda a la evolución favorable de estas patologías.

Palabras clave: Tendinopatía; Entrenamiento excéntrico; Ejercicio para tendinopatía; Ejercicio excéntrico.

\section{ABSTRACT}

Introduction: In 2017, at Mexico City, a statistic about the main pathologies in High Performance Athletes was made. Injury prevalence in athletes was of $58.14 \%$, tendinopathy was the 3rd more frequent injury (about 12.64\%). Based on these findings, the concepts, treatments, and approaches to tendinopathy are review. Here are described eccentric exercises protocols which proved the efficacy of eccentric exercise. The efficacy of this 
kind of exercises is due to the response of tendons to eccentric training. Tendinopathy, collective name for tendinitis, tendinosis and paratendinitis, is a clinic syndrome that describes tendon injuries upon excessive use. Tendinopathy is characterized as a combination of pain, diffuse or localized inflammation and function loss, thermic damage, and adaptive compressive responses. Objective: Through a review of the specialized literature on tendinopathies and their treatment, an attempt will be made to establish the efficacy of eccentric exercise protocols from their conception to their application to tendinopathy conditions and to present the advantage they have over other types of exercises. Methodology: Eccentric exercise protocols that demonstrate the efficacy of eccentric exercise are described. The effectiveness of this type of exercise is due to the response of the tendons to eccentric training. Results: In this literature review, it is reported that well-designed eccentric exercise programs significantly improve tendinopathy patients and results are maintained in the medium to long term. Conclusions: The review of the literature presented here sheds light on the benefits of eccentric exercise. Specifically, in the metabolic process and in the mechanical properties in the tendon, which helps the favorable evolution of these pathologies.

Keywords: Tendinopathy; Eccentric training; Tendinopathy exercise; Eccentric exercise.

\section{INTRODUCCIÓN}

El deporte, en sus múltiples manifestaciones, se ha convertido en una de las actividades sociales con mayor arraigo y capacidad de movilización (CanedoGarcía et al. 2019). Tanto el deporte como la actividad física, en general, han aumentado su consolidación, como actividades esenciales en las últimas décadas, por todo tipo de poblaciones y con un amplio abanico de edades, buscando beneficios, no solo en cuanto al rendimiento sino también en relación con la salud (Torrecillas \& Segura, 2019).

El tendón es un elemento fundamental dentro del aparato locomotor. Su participación es clave durante las actividades deportivas, durante las cuales, se ve sometido a importantes fuerzas, lo que determina que sea una estructura susceptible de lesionarse (Reinking, 2012).

La tendinopatía, nombre colectivo para tendinitis, tendinosis y paratendinitis, es el síndrome clínico, que describe las lesiones por uso excesivo del tendón. Se caracteriza por una combinación de dolor, inflamación difusa o localizada y pérdida de la función, daño térmico y respuestas compresivas adaptativas (Suer \& Abd-Elsayed, 2019). Se trata de una respuesta fallida a la curación, asociada a degeneración de las células tendinosas, desorden de las fibras de colágeno, aumento de la matriz celular sin colágeno junto con una inflamación neurogénica (Scott et al. 2015).

Entre el 1 de mayo de 2017 al 31 de octubre de 2017, en la Ciudad de México, se realizó una estadística sobre las principales patologías en atletas de alto rendimiento, en la cual, se registraron un total de 3.875 deportistas. Fueron considerados los 3.875 deportistas, con un total de 2.253 lesiones reportadas. La prevalencia de lesiones en los deportistas fue de $58,14 \%$, siendo la tendinopatía la tercera patología más frecuente, con un 12,64\% (Tabla 1).

Tabla 1. Estadística realizada en Ciudad de México, con atletas de alto rendimiento.

\begin{tabular}{|c|c|c|}
\hline $\mathbf{n = 2 . 2 5 3}$ & Número de casos & Porcentaje \\
\hline Contractura & 475 & $21,08 \%$ \\
\hline Esguince & 346 & $15,35 \%$ \\
\hline Tendinopatía & 285 & $12,64 \%$ \\
\hline $\begin{array}{c}\text { Síndrome manguito } \\
\text { rotador }\end{array}$ & 135 & $5,99 \%$ \\
\hline Lumbalgia & 131 & $5,81 \%$ \\
\hline
\end{tabular}


Objetivo. A través de una revisión de la literatura especializada sobre tendinopatías y su tratamiento, se buscará establecer la eficacia de los protocolos de ejercicios excéntricos, desde su concepción hasta su aplicación, a padecimientos de tendinopatía y enunciar la ventaja que tienen sobre otros tipos de ejercicios.

\section{MATERIALES Y MÉTODOS}

Diseño. Se realizó la búsqueda de investigaciones que referenciaran la eficacia del ejercicio excéntrico en tendinopatías. Asimismo, se enfatizó en la literatura que hiciera hincapié en programas de ejercicios excéntricos, especialmente, en los que demostraron los resultados más eficaces para la recuperación de la lesión.

Estrategia de búsqueda. Se llevó a cabo, la búsqueda de literatura especializada, con palabras clave, como tendinopatía, ejercicios excéntricos, entrenamiento excéntrico, que tuvieran referencias con el uso de ejercicios y los beneficios que tiene el incluirlos a la recuperación del atleta y los que hablaran de las problemáticas de las tendinopatías; asimismo, artículos actuales y de autores, que tuvieran estudios de los programas ya establecidos.

Criterios de inclusión. De todos los artículos encontrados, se seleccionaron aquellos que hicieran referencia acerca de la tendinopatía, la fisiopatología y los efectos y beneficios de los ejercicios excéntricos, ya reconocidos.

Criterios de exclusión. Se omitieron los artículos que no tuvieran muestra significativa, información fiable o bibliografía, que no estuviera respaldada con estudios recientes.

Extracción de datos. Las bases de datos consultadas fueron Pubmed, HighBeam Research, Redalyc, Chemedia, RefSeek, SciELO, Google Academic.

Fisiopatología. Ante una agresión (tensión, compresión o fricción), que lesiona el tendón, reacciona con una respuesta vascular y fibroblástica, que trata de reparar el daño. Millar et al. (2010) proponen que hay respuesta aparente de las células del sistema inmunitario, responsables de la inflamación. Si el proceso de recuperación no consigue su objetivo, aparecen en zona de tendinosis, que facilitan la cronificación del dolor. El origen del problema parece ser una respuesta incompleta (Gross, 1992); lo que se desconoce es sí, se debe a una sobrecarga excesiva (microlesiones repetidas, que acaban superando la capacidad natural de reparación), a una lesión aguda irreparable (que pudo pasar inadvertida) o un defecto constitucional del tendón de origen genético (Khan et al. 1996; Paavola et al. 2000; Park et al. 2019; Scott et al. 2015).

- Modelo tradicional: Propone que el sobreuso del tendón provoca inflamación y, por lo tanto, dolor (Maffulli et al. 2008).

- Modelo mecánico: Atribuye el dolor a dos situaciones: por un lado, a una lesión de las fibras de colágeno, aunque existen situaciones en las que el tendón está completamente intacto y también hay dolor. Una variante de esta teoría afirma que no es la rotura de colágeno lo que produce dolor, sino el colágeno intacto residual contiguo al lesionado, debido al estrés añadido, que supera su capacidad normal de carga (Jurado \& Medina-Porqueres, 2008).

- Modelo bioquímico: Propone que la causa del dolor es una irritación química, debida a una hipoxia regional y a la falta de células fagocitarias, para eliminar productos nocivos de la actividad celular.

- Modelo vasculonervioso: Se basa en el daño neural y la hiperinervación, sugiere que las fibras nerviosas positivas para la sustancia $P$ se encuentran localizadas en la unión hueso-periostio-tendón (Barcelona, 2012).

Etiología. El origen de las lesiones tendinosas tiene una relación directa con el tipo de fuerza que actúa sobre el tendón. Las lesiones tendinosas son producidas, principalmente, por fuerzas de compresión, fuerzas de rozamiento o fricción, fuerzas de tracción o por diferentes estímulos de leve intensidad aplicados de forma repetitiva (Tabla 2). Es por ello, que estas lesiones pueden tener su origen en factores intrínsecos o en factores extrínsecos (Barcelona, 2012) (Tablas 3 y 4). 
Tabla 2. Clasificación de tendinopatías.

\begin{tabular}{|l|l|}
\hline Diagnóstico & Hallazgos microscópicos \\
\hline Tendinosis & $\begin{array}{l}\text { Degeneración tendinosa causada por la edad, el } \\
\text { envejecimiento del tejido conjuntivo, el sobreuso y el } \\
\text { compromiso vascular }\end{array}$ \\
\hline $\begin{array}{l}\text { Tendinopatía/rotura } \\
\text { parcial }\end{array}$ & Degeneración sintomática con disrupción vascular \\
\hline Paratendinitis & $\begin{array}{l}\text { Inflamación del paratendón, sin tener en cuenta si está } \\
\text { cubierto o no de sinovial }\end{array}$ \\
\hline $\begin{array}{l}\text { Paratendinitis con } \\
\text { tendinosis }\end{array}$ & $\begin{array}{l}\text { Paratendinitis asociada con degeneración } \\
\text { intratendinosa }\end{array}$ \\
\hline
\end{tabular}

Tomado de Futbol Club Barcelona, Serveis Médics (2012).

Tabla 3. Factores intrínsecos asociados a la tendinopatía.

\begin{tabular}{|c|c|}
\hline Generales & Locales \\
\hline $\begin{array}{l}\text { Sexo } \\
\text { Edad }\end{array}$ & $\begin{array}{l}\text { Malalineaciones: Pie hiperpronado o hipopronado, } \\
\text { genuvalgo/varo, anteversión del cuello femoral } \\
\text { Dismetría de miembros inferiores (MM.II.) } \\
\text { Debilidades musculares } \\
\text { Desequilibrios musculares } \\
\text { Laxitud articular } \\
\text { Disminución de la flexibilidad }\end{array}$ \\
\hline
\end{tabular}

Factores intrínsecos (Paavola et al. 2005).

Tabla 4. Factores extrínsecos asociados a la tendinopatía.
Métodos de entrenamiento
Duración o intensidad excesiva
Déficit de adaptación fisiológica
Inadaptación a la especificidad del entrenamiento
Incrementos súbitos en el programa de entrenamiento
Errores en la adaptación individual al entrenamiento
Cambios de superficie de entrenamiento/juego Calentamiento
insuficiente
Entrenamiento general inadecuado
Recuperación insuficiente
Problemas derivados del material

Factores extrínsecos (Paavola et al. 2005). 
Diagnóstico. Las pruebas más utilizadas para el diagnóstico de tendinopatía son la Resonancia Magnética (RM) y la ultrasonografía (US) (Bley \& Abid, 2015; Millar et al. 2010; Ruaro et al. 2019).
La clasificación clínica más utilizada para la valoración de esta lesión es la escala de Blazina (Blazina et al. 1973) (Tabla 5), que se basa en criterios de evolución del dolor, según la funcionalidad.

Tabla 5. Clasificación del dolor de Blazina et al. (1973).

\begin{tabular}{|l|l|}
\hline Estadio 1 & $\begin{array}{l}\text { El dolor solo aparece después de la actividad deportiva y no influye } \\
\text { en el rendimiento del deportista }\end{array}$ \\
\hline Estadio 2 & $\begin{array}{l}\text { El atleta presenta dolor en el inicio de la actividad deportiva, pero } \\
\text { con el calentamiento desaparece y tampoco afecta, de forma } \\
\text { significativa, el rendimiento deportivo }\end{array}$ \\
\hline Estadio 3 & $\begin{array}{l}\text { El dolor está presente durante y después de la actividad deportiva, } \\
\text { provocando, incluso, que el deportista se vea obligado a dejar su } \\
\text { actividad deportiva }\end{array}$ \\
\hline Estadio 4 & Rotura del tendón \\
\hline
\end{tabular}

Tratamiento. La primera opción es seguir las recomendaciones de autores, como Nirschl (1992), Puddu et al. (1976), Stanish et al. (1986), quienes, desde hace más de 20 años, han hablado y siguen hablando, de los conceptos de tendinosis y desorganización del colágeno. Estos autores proponen aliviar, inicialmente el dolor, con las medidas clásicas (frío, analgésicos o AINE, ocasionalmente infiltración con corticoides o medidas físicas), pero a lo que dan la máxima importancia es a promover la curación del tendón fortaleciéndolo mediante programas de ejercicios activos (Stevens \& Tan, 2014). El concepto clave para estos autores es que, debido a la integridad funcional de la unidad músculo-tendón, el ejercicio también influye sobre el tendón.

La segunda opción y que constituye, actualmente, un área muy activa de investigación es identificar los factores bioquímicos, que pueden influir o ser responsables del fallo en el proceso de reparación tendinosa y emplearlos con fines terapéuticos. Se ha propuesto la utilización del factor transformante de crecimiento beta (una sustancia que participa en los procesos reparadores de nuestro organismo), la inyección de células madre o la terapia genética (Ackermann et al. 2018; Andriolo et al. 2019)

Programa de ejercicios excéntricos. Con relación a las tendinopatías, se ha incorporado el trabajo excéntrico, como medio de prevención y de recuperación de dicha lesión. Las tendinopatías más habituales son tendinopatía aquilea, tendinopatía rotuliana y tendinopatía en los epicondilos medial y lateral del codo (Cannel et al. 2001).

La base del movimiento y el ejercicio es la contracción muscular. Habitualmente, se considera la contracción muscular como un acortamiento del músculo que ocurre cuando los filamentos de actina y miosina se deslizan unos sobre otros; sin embargo, en las actividades cotidianas, la longitud del músculo no cambia (Garnier et al. 2019) y, en algunos casos, puede aumentar. Estas diferencias en el comportamiento del músculo durante el ejercicio han conducido a clasificar la contracción muscular (o el ejercicio) en isométrico (sin cambio de longitud) (Holden et al. 2020), isotónico (donde hay desplazamiento articular y se puede clasificar en concéntrico (con acortamiento de fibras) (Paulus et al. 2019) y excéntrico (el músculo se alarga, mientras se produce la fuerza) (Roig et al. 2009). Las contracciones excéntricas son muy importantes en nuestras actividades cotidianas y deportivas y van a ser un elemento importante en los programas de ejercicios terapéuticos en tendinopatías (Cheng et al. 2005; Vallejo et al. 2006).

Los estudios en los que se aplica un trabajo excéntrico para tratar las tendinopatías indican los beneficios de este tipo de tratamiento respecto a otros. Mediante pruebas clínicas es contrastado con diferentes terapias o tipos de ejercicios (Andriolo et al. 2019; Dejaco et al. 2017; Dimitrios, 2017; Jayaseelan et al. 2019; Stefansson et al. 2019; Sussmilch-Leitch et al. 2012; van Rijn et al. 2019). 
La causa por la que este tipo de tratamiento es eficaz, se debe a la respuesta de los tendones al entrenamiento excéntrico, ya que aumentan la actividad metabólica y el tamaño de los vasos sanguíneos (Knobloch et al. 2007), que tiene como consecuencia una intensificación de la síntesis de colágeno (Heinemeier et al. 2003; Langberg, 2013), provocando una hipertrofia y mejora de las propiedades mecánicas del tendón (Arampatzis et al. 2007; Kongsgaard et al. 2007; Seynnes et al. 2009).

Programa de ejercicios en tendinopatía aquilea. Entre las modalidades de tratamiento conservador de las tendinopatías de Aquiles, muchos autores han incluido los programas de ejercicios (Cardoso et al. 2019; El Hawary et al. 1997; Sussmilch-Leitch et al. 2012).

El primer trabajo que sugiere la superioridad de este tipo de ejercicios, se publica en 1992. NiesenVertommen et al. (1992) observan que, en pacientes con tendinopatía del Aquiles de más de 4 semanas de evolución, un programa de ejercicios excéntricos mejoraba más rápidamente los síntomas, que un programa de ejercicios concéntricos.
Los dos estudios más importantes y de mayor calidad metodológica, prospectivos, aleatorizados y con grupo control, no se publican hasta el 2001 (Cannell et al. 2001; Croisier et al. 2001). En ambos estudios, solo se incluyen pacientes con aquilodinia proximal (dolor y sensibilidad dolorosa en la parte media del tendón, a $2-6 \mathrm{~cm}$ de la inserción, a menudo con un nódulo palpable), el tipo más habitual en deportistas (O'Neill et al. 2019).

El tratamiento mediante ejercicios excéntricos ha presentado resultados esperanzadores y muy positivos a corto y medio plazo (Sussmilch-Leitch et al. 2012). En estudios realizados con pacientes que sufrían tendinopatía 2-6 centímetros por encima del nivel de su inserción con el calcáneo, pero no en aquellos a quienes el dolor del tendón, se presentaba justo a nivel de inserción, a diferencia del tratamiento de régimen concéntrico (Flórez et al. 2003). La eficacia del protocolo Alfredson (Tabla 6), para el tratamiento de tendinopatía aquílea está probada y es la que mayores beneficios presenta (Stevens \& Tan, 2014).

Tabla 6. Implementación de ejercicios excéntricos del protocolo Alfredson.

Estos ejercicios se prescriben 2 veces diarias, 7 días a la semana, durante 12
semanas.
El paciente parte de la flexión
plantar con los dos pies, realiza
la fase excéntrica de bajada con
un solo pie hacia la flexión
dorsal. Vuelve a subir y se pone
de puntillas, fase concéntrica,
con los dos pies.
Mismo ejercicio. El paciente
parte de la flexión plantar con
ambos pies y realiza la fase
excéntrica de bajada. Sigue con
la rodilla flexionada y con un solo
pie hacia la flexión dorsal. El
paciente sube la pierna y se
pone de puntillas, fase
concéntrica, con los dos pies.


Continuación tabla 6.

Pediremos al paciente que se
coloque al borde de un escalón,
apoyando sólo el antepié y deja
libre el retropié de la extremidad
lesionada (apoyo unipodal).
Hará una flexión dorsal máxima
del tobillo, controlada y lenta.
Después, hará el excéntrico del
tríceps, partiendo desde la
flexión plantar máxima (se
puede poner peso dentro de la
mochila).
Este ejercicio se indica en la fase
3 y se deben realizar 3 series de
15 repeticiones.
La misma posición del ejercicio
anterior. Cuando el paciente
realice el ejercicio sin molestia, o
note que ésta desciende,
añadiremos peso. Si fuera
necesario utilizar grandes pesos,
se permite utilizar máquinas de
gimnasio.
Este ejercicio se indica en la fase
3 y se debe realizar 3 series de
15 repeticiones

Programa de ejercicios en tendinopatía rotuliana. Los programas de fortalecimiento muscular, se consideran, actualmente, el elemento clave en el tratamiento de estas tendinopatías (Cook et al. 2000). Curwin \& Stanish (1984) proponen un programa de ejercicios excéntricos durante 6-8 semanas, con el que observan un alivio completo de los síntomas en un tercio de los casos y mejoría parcial en los dos tercios restantes. En esta misma década, se publican dos ensayos clínicos, de mala calidad metodológica, que describían una mejoría en el dolor (Cannell, 1982) y en la fuerza muscular utilizando un programa similar, al descrito por Curwin \& Stanish (1984) (Jensen \& Di Fabio, 1989). En 1992, Karlsson et al. (1992) publican excelentes resultados en el $70 \%$ de 81 pacientes, con un protocolo de rehabilitación, que combinaba ejercicios de fortalecimiento muscular concéntrico y excéntrico. A partir de entonces, el único ensayo clínico prospectivo, aleatorizado y controlado no se publica hasta el año (Cannell et al. 2001). Compara un programa de ejercicios excéntricos con cargas progresivas durante 12 semanas, con un programa de fortalecimiento muscular concéntrico estándar (elevación del miembro inferior recto e isotónicos de isquiotibiales en decúbito prono, colocando pesos en el tobillo de forma progresiva). La mayor parte de los pacientes mejoraron, pero, especialmente, los que realizaron el programa de ejercicios excéntricos: 9 de 10 volvieron a su actividad deportiva habitual, frente a 6 de 9, en el programa de ejercicios concéntricos (Tabla 7). 
Tabla 7. Ejercicios excéntricos.

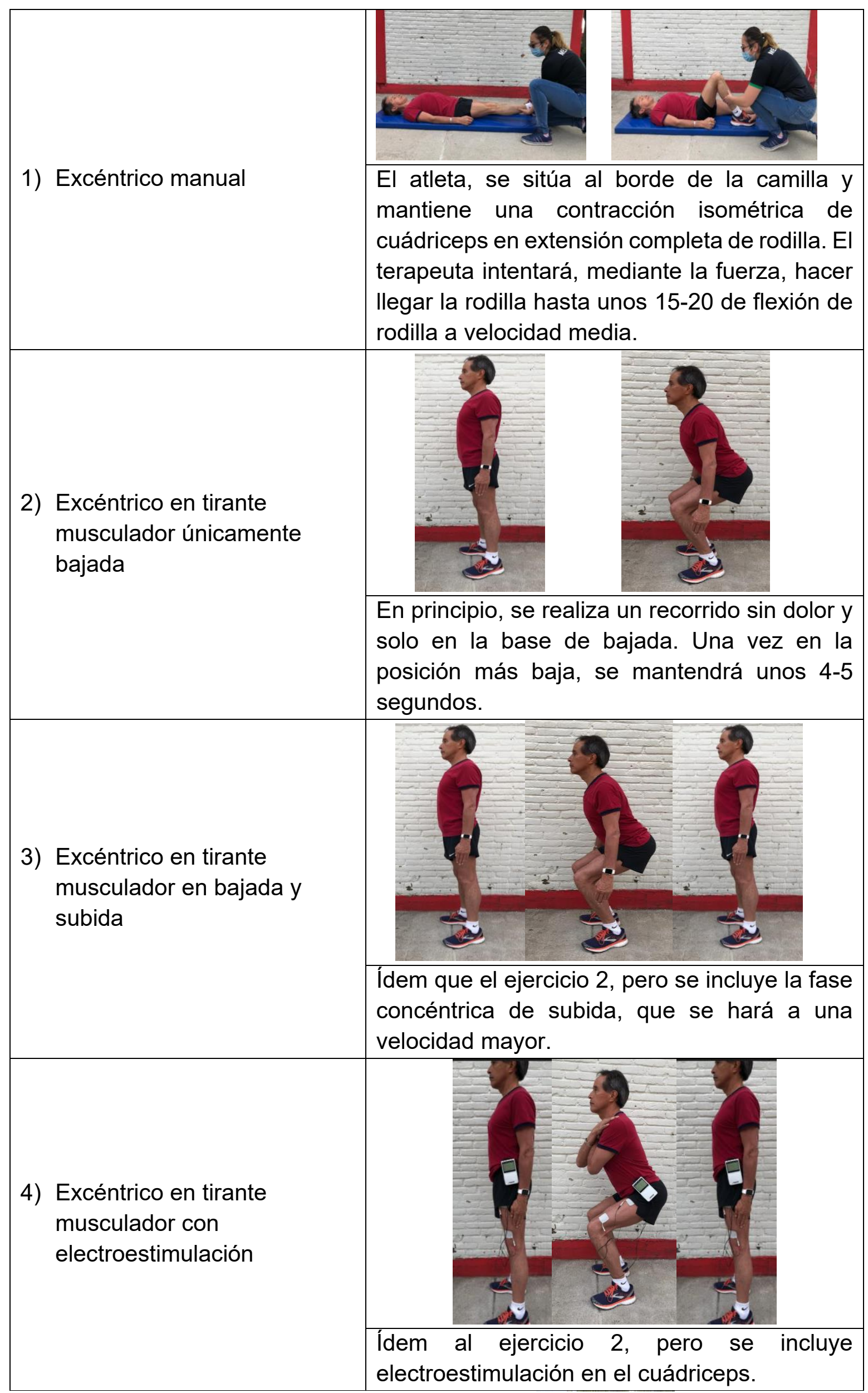


Continuación tabla 7.

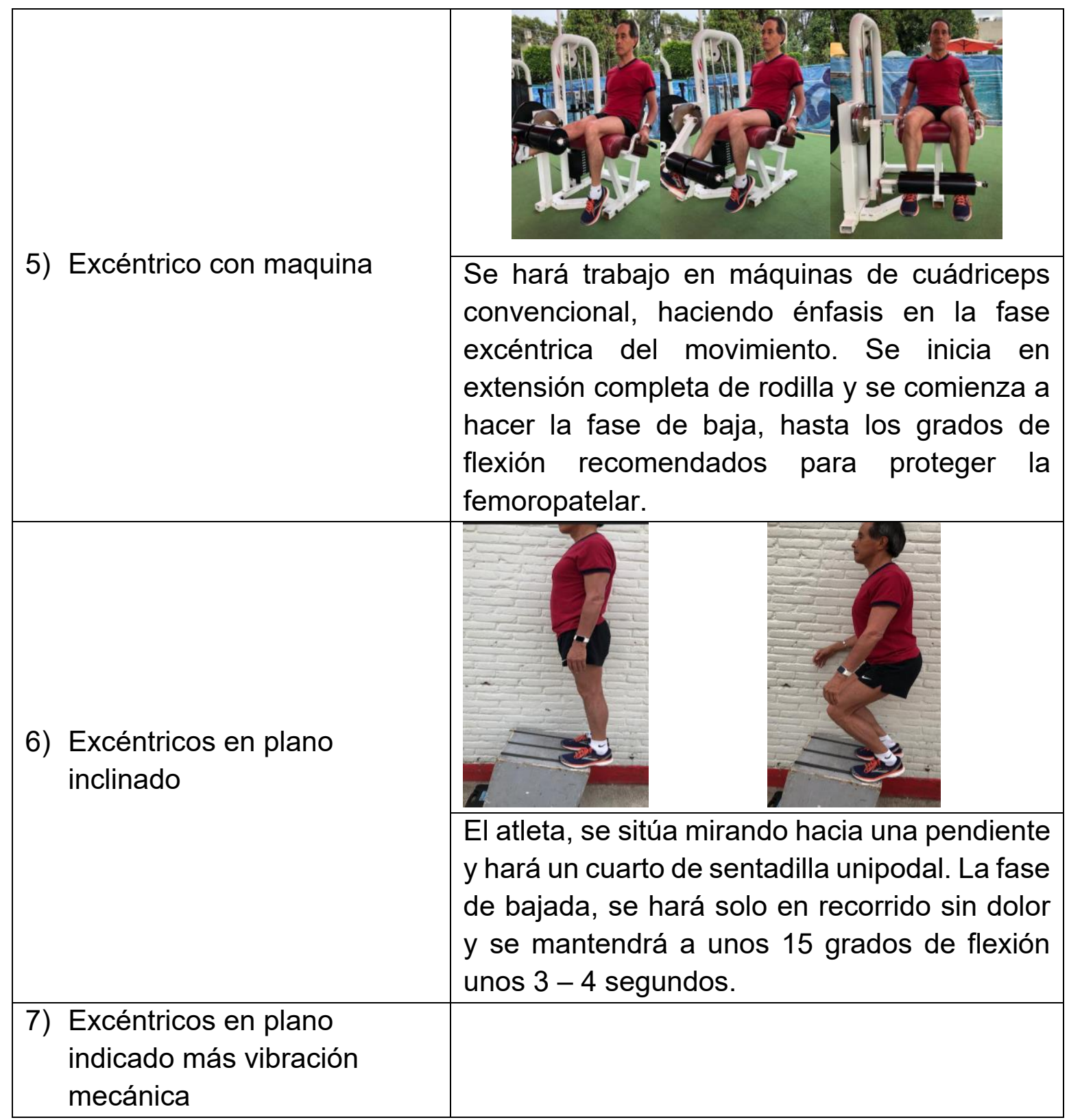

Programa de ejercicios excéntricos en epicondilitis. Aunque el protocolo propuesto por Fillion (1991) (Tabla 8), menciona los programas de ejercicios activos, como tratamiento único de la epicondilitis, habitualmente, los ejercicios de fortalecimiento muscular solo se recomendaban cuando el dolor se había controlado con otras medidas (medicación, medios físicos, infiltración y otros) (Hong et al. 2003;
Tarpada et al. 2018) y su objetivo principal era preparar al paciente, para reanudar su actividad laboral o deportiva. Con el ejercicio, se pretendía recuperar una musculatura que se había debilitado por un largo proceso de inactividad secundario al dolor. En la fase aguda, solo se indicaban ejercicios pasivos de estiramiento, realizados suavemente y siempre evitando provocar dolor. 
Tabla 8. Protocolo de Fillion para epicondilitis (Fillion, 1991).

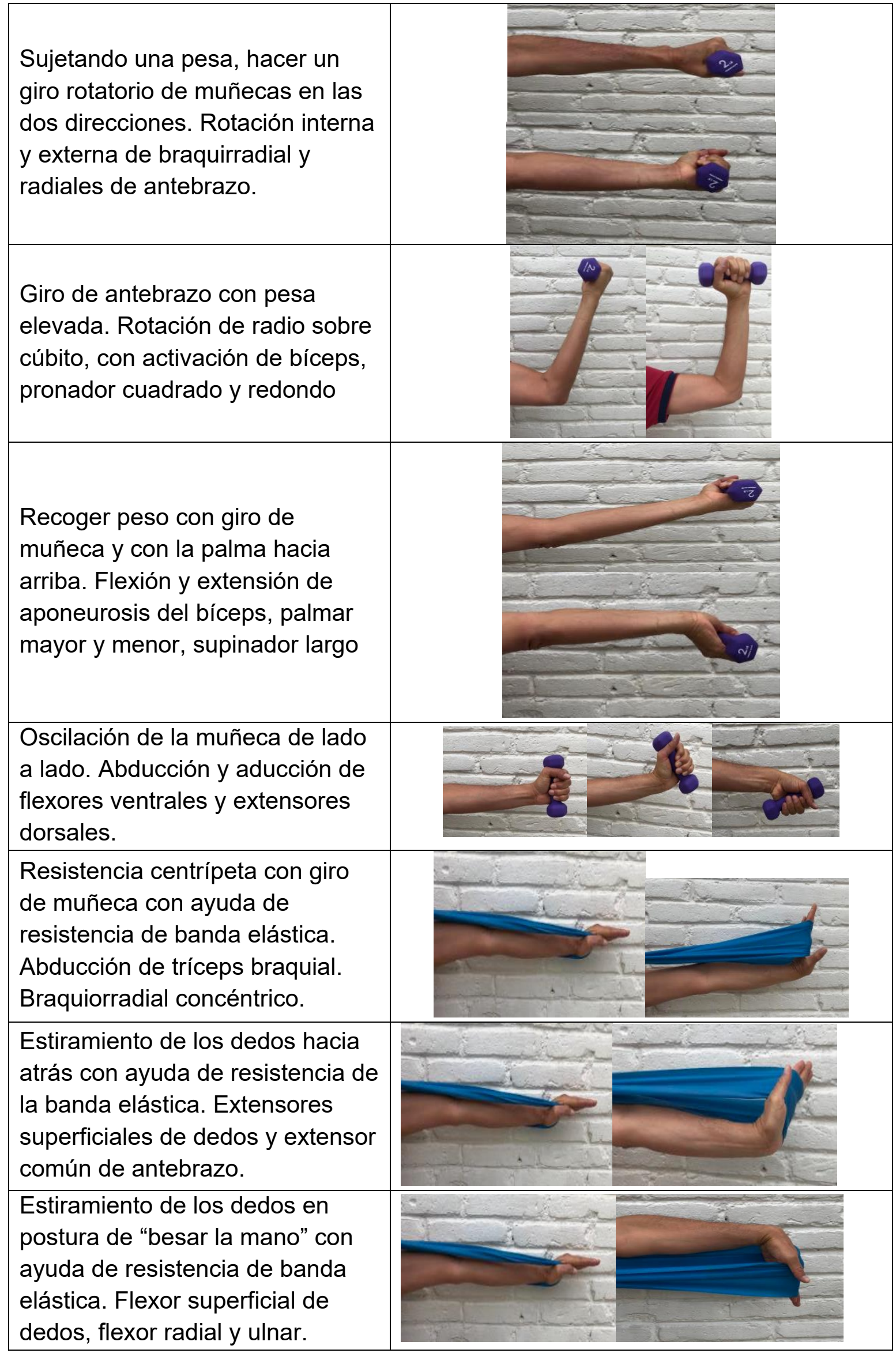


Continuación tabla 8.

\begin{tabular}{|c|c|}
\hline $\begin{array}{l}\text { Estiramiento de los dedos } \\
\text { lateralmente con ayuda de } \\
\text { resistencia de banda elástica. } \\
\text { Extensor digital lateral y } \\
\text { ligamento transverso dorsal del } \\
\text { carpo. }\end{array}$ & 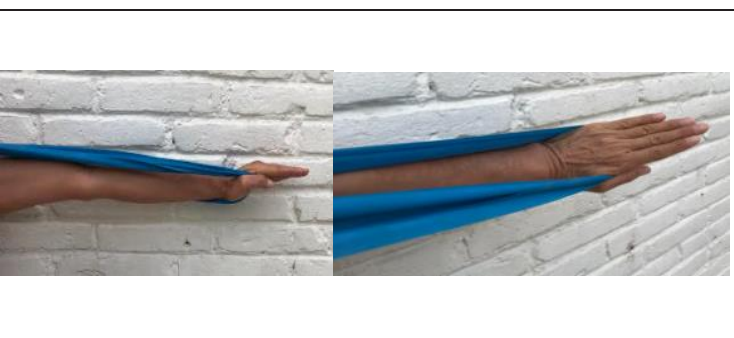 \\
\hline $\begin{array}{l}\text { Estiramiento de palma hacia } \\
\text { atrás con flexión de dedos con } \\
\text { ayuda de resistencia de banda } \\
\text { elástica. Extensor epicóndilo } \\
\text { medial, palmar largo y flexor del } \\
\text { carpo (nervio medial) y extensor } \\
\text { común de los dedos. }\end{array}$ & \\
\hline
\end{tabular}

Actualmente, se evalúan alternativas para el tratamiento de la epicondilitis (Bostrøm et al. 2019; Mautner et al. 2011; Tarpada et al. 2018). Las evidencias sobre métodos regenerativos para el tendón, a través de Plasma Rico, en Plaquetas y Concentrado de Aspirado de Médula Ósea, tienen resultados; sin embargo, el tratamiento con ejercicios es todavía el que mejores resultados aporte (Bostrøm et al. 2019; Davies, 2003).

\section{RESULTADOS Y DISCUSIÓN}

Los ejercicios excéntricos son una herramienta muy importante dentro de la rehabilitación, ya que la teoría plantea que disminuyen el dolor más rápido que los estiramientos, el fortaleciendo y el endureciendo el tendón. Gómez Díaz (2016) refiere que uno de los estudios más importantes y que ha demostrado mayor eficacia de los ejercicios excéntricos son los de Alfredson, en donde plantea tres posibles teorías, que explican su efectividad. La primera teoría plantea que se da una alteración en la percepción del dolor por parte del atleta, debido a que los ejercicios pueden llegar a ser dolorosos, Mchug ha indicado que tras un entrenamiento excéntrico se observa una pérdida de fuerza explosiva durante periodos superiores a 24 horas (principalmente, en personas o deportistas que no están acostumbrados al mismo) y la posibilidad de inducir daño muscular, si el ejercicio no se realiza de la manera efectiva (Mchug et al. 2000). La segunda teoría de Alfredson explica que, al realizar el trabajo excéntrico se destruye la vascularización que aparece en la tendinopatía y, con ello, las terminaciones nerviosas que la acompañan y la tercera teoría de Alfredson refiere que, cuando se ejerce un ejercicio excéntrico aumenta la resistencia a la tracción del tendón, lo que produce una elongación de la unidad musculo tendinosa, dando la capacidad de soportar menores tensiones durante el movimiento. Siguiendo esta línea, Kjaer et al. (2005) refieren que un programa de ejercicio excéntrico afecta a la producción de colágeno tipo I y en ausencia de actividades agresivas paralelas, puede aumentar el volumen del tendón a largo plazo.

En la actualidad, hay diversos estudios con base en los principales programas de ejercicios excéntricos, donde hay diversas explicaciones, que conducen a entender la efectividad del uso de este tipo de ejercicios, aunque no están investigadas en su totalidad; los resultados del entrenamiento excéntrico han demostrado una mejoría significativamente, en cuanto a la disminución del dolor y la funcionalidad del tendón en el deportista, de un 60-90\% (Schmikli et al. 2009).

Esta revisión bibliográfica sostiene que, con base en los resultados obtenidos y reportados por Alfredson et al. (1999), Curwin \& Stanish (1984) y Fillion (1991) los programas de ejercicios excéntricos bien diseñados mejoran significativamente a los atletas con tendinopatías y los resultados se mantienen a medio-largo plazo, considerándolos como una de las principales opciones de tratamiento, en atletas de alto rendimiento.

Recomendaciones prácticas. El modelo de entrenamiento excéntrico no implica, en ningún momento, una contracción concéntrica del tendón afectado y hace especial mención a la importancia de realizar los ejercicios, aunque el paciente tenga alguna molestia, siempre y cuando permita esa molestia 
realizar el ejercicio correctamente. Si los pacientes no experimentan ningún dolor ni molestia durante la realización del ejercicio pueden aumentar la carga, mediante una mochila con peso o con máquinas de musculación. El entrenamiento excéntrico consiste en un entrenamiento diario durante 12 semanas, donde se obtiene, al término, buenos resultados a largo y corto plazo (Gómez Díaz 2016).

El entrenamiento excéntrico, se debe ejercer de forma progresiva, con relación al medio ambiente, teniendo en cuenta los aspectos generales y específicos de la disciplina deportiva de cada atleta. El incremento progresivo, la dificultad del ejercicio y las pautas adecuadas (\# de repeticiones, \# de series, \# de ejercicios, \% de carga, velocidad), estarán influenciados por las características de cada sujeto (umbral de dolor, umbral de entrenamiento, capacidad muscular, tiempo de recuperación de la lesión. Asimismo, se debe de evitar la inmovilización completa del tendón durante el entrenamiento, sugerido por Khan et al. (2000), quien explica que la inmovilización completa de un tendón lesionado está contraindicada, ya que la tensión de carga estimula directamente la producción de colágeno y su alineación.

\section{CONCLUSIÓN}

Como conclusión provisional, aún no se cuenta con una respuesta definitiva sobre otras alternativas a los ejercicios excéntricos en el tratamiento de tendinopatías. Los estudios y las opiniones de los expertos no dan una respuesta final (Cardoso et al. 2019; Cook et al. 2018; Jayaseelan et al. 2019; Stergioulas et al. 2008); sin embargo, la revisión de la literatura que aquí se presenta arroja luz sobre los beneficios que tienen los ejercicios excéntricos, especialmente, en el proceso metabólico y en las propiedades mecánicas en el tendón, lo cual, de manera específica, ayuda a la evolución favorable de estas patologías (Grävare Silbernagel et al. 2011).

\section{REFERENCIAS}

1. ACKERMANN, P.; PHISITKUL, P.; PEARCE, C. 2018. Treatment of Achilles tendinopathy: state of the art. Journal of ISAKOS: Joint Disorders \& Orthopaedic Sports Medicine. 3(6):367-376. https://doi.org/10.1136/jisakos-2018-000202

2. ANDRIOLO, L.; ALTAMURA, S.; REALE, D.; CANDRIAN, C.; ZAFFAGNINI, S.; FILARDO,
G. 2019. Nonsurgical Treatments of Patellar Tendinopathy: Multiple Injections of PlateletRich Plasma Are a Suitable Option: A Systematic Review and Meta-analysis. American Journal of Sports Medicine. 47(4):1001-1018. https://doi.org/10.1177/0363546518759674

3. ALFREDSON H, THORSEN K, LORENTZON R. 1999. In situ microdialysis in tendon tissue: high levels of glutamate, but not prostaglandin E2 in chronic Achilles tendon pain. Knee Surg Sports Traumatol Arthrosc. 7:378-81.

4. ARAMPATZIS, A.; KARAMANIDIS, K.; ALBRACHT, K. 2007. Adaptational responses of the human Achilles tendon by modulation of the applied cyclic strain magnitude. The Journal of Experimental Biology. 210:2743-2753. https://doi.org/10.1242/jeb.003814

5. BARCELONA, F.C. 2012. Guía de la práctica clínica de les tendinopaties: diagnóstic, tractament i prevenció. Apunts. Medicina de l'Esport. 47(176):143-168.

https://doi.org/10.1016/j.apunts.2012.09.001

6. BLAZINA, M.; KERLAN, R.; JOBE, F.; CARTER, V.; CARLSON, G. 1973. Jumper's knee. The Orthopedic Clinics of North America. 4(3):665-678.

7. BLEY, B.; ABID, W. 2015. Imaging of tendinopathy: A physician's perspective. Journal of Orthopaedic and Sports Physical Therapy. 45(11):826-828.

https://doi.org/10.2519/jospt.2015.0113

8. BOSTRØM, K.; MAEHLUM, S.; SMÅSTUEN, M.; STORHEIM, K. 2019. Clinical comparative effectiveness of acupuncture versus manual therapy treatment of lateral epicondylitis: Feasibility randomized clinical trial. Pilot and Feasibility Studies. 5(1):1-10. https://doi.org/10.1186/s40814-019-0490-x

9. CANEDO-GARCÍA, A.; PACHECO-SANZ, D.I.; GARCÍASÁNCHEZ, J.N. 2019. La actividad físicodeportiva y los programas intergeneracionales. In: Padilla, D.; Aguilar, J.; López, R. (eds). Salud y Ciclo Vital. 1st ed. Dykinson, S.L. p.319-331. https://doi.org/10.2307/j.ctvfb6z2h.31

10. CANNELL, L. 1982. The effects of an eccentric-type exercise versus a concentric-type exercise in the management of chronic patellar tendonitis. The University of British Columbia. 70p. 
11. CANNELL, L.; TAUNTON, J.; CLEMENT, D.; SMITH, C., KHAN, K. 2001. A randomised clinical trial of the efficacy of drop squats or leg extension/ leg curl exercises to treat clinically diagnosed jumper's knee in athletes: Pilot study. British Journal of Sports Medicine. 35(1):60-64. https://doi.org/10.1136/bjsm.35.1.60

12. CARDOSO, T.; PIZZARI, T.; KINSELLA, R.; HOPE, D.; COOK, J. 2019. Current trends in tendinopathy management. Best Practice and Research: Clinical Rheumatology. 33(1):122-140. https://doi.org/10.1016/j.berh.2019.02.001

13. CHENG, H.;WU, S.; KAO, M. 2005. Cardiopulmonary responses to isokinetic concentric/eccentric exercise of the quadriceps in healthy young adults. 10:S28-S36.

14. COOK, J.; KHAN, K.; MAFULLI, N.; PURDAM, C. 2000. Overuse Tendinosis, Not Tendinitis Part 2: Applying the New Approach to Patellar Tendinopathy. The Physician and Sportsmedicine. 28(6):31-46. https://doi.org/10.1080/00913847.2000.11 439512

15. COOK, J.; STASINOPOULOS, D.; BRISMÉE, J.M. 2018. Insertional and mid-substance Achilles tendinopathies: eccentric training is not for everyone-updated evidence of nonsurgical management. Journal of Manual and Manipulative Therapy. 26(3):119-122.

https://doi.org/10.1080/10669817.2018.147 0302

16. CROISIER, J.; FORTHOMME, B.; FOIDARTDESALLE, M.; GODON, B.; CRIELAARD, J. 2001. Treatment of recurrent tendinitis by isokinetic eccentric exercises. Isokinetics and Exercise Science. 9(2-3):133-141.

https://doi.org/10.3233/IES-2001-0077

17. CURWIN, S.; STANISH, W. 1984. Tendinitis: its etiology and treatment. Collamore Press. $189 p$.

18. DAVIES, C. 2003. Self-treatment of lateral epicondylitis (tennis elbow): Trigger point therapy for triceps and extensor muscles. Journal of Bodywork and Movement Therapies. 7(3):165-172. https://doi.org/10.1016/S13608592(02)00071-2
19. DEJACO, B.; HABETS, B.; VAN LOON, C.; VAN GRINSVEN, S.; VAN CINGEL, R. 2017. Eccentric versus conventional exercise therapy in patients with rotator cuff tendinopathy: a randomized, single blinded, clinical trial. Knee Surgery, Sports Traumatology, Arthroscopy. 25(7):2051-2059.

https://doi.org/10.1007/s00167-016-4223-x

20. DIMITRIOS, S. 2017. The effectiveness of eccentric - concentric training and isometric contractions on pain and disability in Achilles tendinopathy. A case report. Physical Therapy in Sport. 28:e3.

https://doi.org/10.1016/j.ptsp.2017.08.014

21. EL HAWARY, R.; STANISH, W.; CURWIN, S. 1997. Rehabilitation of tendon injuries in sport. Sports Medicine. 24:347-358. https://doi.org/10.2165/00007256199724050-00006

22. FILLION, P.1991. Treatment of Lateral Epicondylitis. American Journal of Occupational Therapy. 45(4):340-343. https://doi.org/10.5014/ajot.45.4.340

23. FLÓREZ, M.; ECHAVARRI, C.; PAVÓN DE PAZ, M. 2003. Programas de ejercicios en tendinopatías. Rehabilitación. 37(6):354-362. https://doi.org/10.1016/s00487120(03)73406-9

24. FUTBOL CLUB BARCELONA, SERVEIS MÈDICS. 2012. Guía de práctica clínica de las tendinopatías: diagnóstico. Apunts: Medicina de l'esport. 47(176):143-68.

25. GARNIER, Y.; PAIZIS, C.; LEPERS, R. 2019. Corticospinal changes induced by fatiguing eccentric versus concentric exercise. European Journal of Sport Science. 19(2):166-176. https://doi.org/10.1080/17461391.2018.14 97090

26. GÓMEZ DÍAZ, J. 2016. Eficacia de los ejercicios excéntricos en tendinopatías rotulianas. Revisión bibliográfica. Archivos de Medicina Del Deporte. 33(1):59-66.

27. GRÄVARE SILBERNAGEL, K.; BRORSSON, A.; LUNDBERG, M. 2011. The majority of patients with Achilles tendinopathy recover fully when treated with exercise alone: A 5-year followup. American Journal of Sports Medicine. 39(3):607-613.

https://doi.org/10.1177/0363546510384789 
28. GROSS, M. 1992. Chronic Tendinitis: Pathomechanics of Injury, Factors Affecting the Healing Response, and Treatment. Journal of Orthopaedic \& Sports Physical Therapy. 16(6):248-261.

https://doi.org/10.2519/jospt.1992.16.6.248

29. HEINEMEIER, K.; LANGBERG, H.; OLESEN, J.; KJAER, M. 2003. Role of TGF-B1 in relation to exercise-induced type I collagen synthesis in human tendinous tissue. Journal of Applied Physiology. 95:2390-2397.

https://doi.org/10.1152/

japplphysiol.00403.2003

30. HOLDEN, S.; LYNG, K.; GRAVEN-NIELSEN, T.; RIEL, H.; OLESEN, J.; LARSEN, L.; RATHLEFF, $M$. 2020. Isometric exercise and pain in patellar tendinopathy: A randomized crossover trial. Journal of Science and Medicine in Sport. 23(3):208-214.

https://doi.org/10.1016/j.jsams.2019.09.015

31. HONG, Q.; DURAND, M.; LOISEL, P. 2003. Treatment of lateral epicondylitis: where is the evidence? Joint Bone Spine. 71(5):369-373.

https://doi.org/10.1016/S1297-

319X(03)00167-2

32. JAYASEELAN, D.; MISCHKE, J.; STRAZZULLA, R. 2019. Eccentric exercise for Achilles tendinopathy: A narrative review and clinical decision-making considerations. Journal of Functional Morphology and Kinesiology. 4(2):34.

https://doi.org/10.3390/jfmk4020034

33. JENSEN, K.; DI FABIO, R. 1989. Evaluation of Eccentric Exercise in Treatment of Patellar Tendinitis. Physical Therapy. 69(3):211-216. https://doi.org/10.1093/ptj/69.3.211

34. JURADO, A.; MEDINA-PORQUERES, I. 2008. Tendón: valoración y tratamiento en fisioterapia. Editorial Paidotribo. 598p.

35. KARLSSON, J.; KÄLEBO, P.; GOKSÖR, L.; THOMEE, R.; SWÄRD, L. 1992. Partial rupture of the patellar ligament. The American Journal of Sports Medicine. 20:390-395.

https://doi.org/10.1177/036354659202000404

36. KHAN, K.; BONAR, F.; DESMOND, P.; COOK, J.; YOUNG, D.; VISENTINI, P.; FEHRMANN, M.; KISS, Z.; O'BRIEN, P.; HARCOURT, P.; DOWLING, R.; O'SULLIVAN, R.; CRICHTON, K.;
TRESS, B.; WARK, J. 1996. Patellar tendinosis (jumper's knee): Findings at histopathologic examination, US, and MR imaging. Victorian Institute of Sport Tendon Study Group. Radiology. 200:821-827.

https://doi.org/10.1148/

radiology.200.3.8756939

37. KHAN, K.M.; COOK, J.L.; TAUNTON, J.E.; BONAR, F. 2000. Overuse tendinosis, not tendinitis part 1: a new paradigm for a difficult clinical problem. Phys Sportsmed. 28(5):38-48. https://doi.org/10.3810/psm.2000.05.890

38. KNOBLOCH, K.; KRAEMER, R.; JAGODZINSKI, M.; ZEICHEN, J.; MELLER, R.; VOGT, P. 2007. Eccentric training decreases paratendon capillary blood flow and preserves paratendon oxygen saturation in chronic achilles tendinopathy. Journal of Orthopaedic and Sports Physical Therapy. 37(5):269-276. https://doi.org/10.2519/jospt.2007.2296

39. KONGSGAARD, M.; REITELSEDER, S.; PEDERSEN, T.; HOLM, L.; AAGAARD, P.; KJAER, M.; MAGNUSSON, S. 2007. Region specific patellar tendon hypertrophy in humans following resistance training. Acta Physiologica (Oxford, England). 191:111-121.

https://doi.org/10.1111/j.1748-

1716.2007.01714.x

40. LANGBERG, H. 2013. Tendinopathy: more questions than answers. British Journal of Sports Medicine. 47(9):e2.

https://doi.org/10.1136/

bjsports-2013-092459.18

41. MAFFULLI, N.; WALLEY, G.; SAYANA, M.; LONGO, U.; DENARO, V. 2008. Eccentric calf muscle training in athletic patients with Achilles tendinopathy. Disability and Rehabilitation. 30(20-22):1677-1684.

https://doi.org/10.1080/09638280701786427

42. MAUTNER, K.; MALANGA, G.; COLBERG, R. 2011. Optimization of ingredients, procedures and rehabilitation for platelet-rich plasma injections for chronic tendinopathy. Pain Management. 1(6):523-532.

https://doi.org/10.2217/pmt.11.56

43. MCHUGH, M.P.; CONNOLLY, D.A.; ESTON, R.G.; GLEIM, G.W. 2000. Electromyographic analysis of exercise resulting in symptoms of muscle damage. Journal of Sports Sciences. 18:163-172. 
44. MILLAR, N.; HUEBER, A.; REILLY, J.; YINGHUA, XU.; FAZZI, U.; MURRELL, G.; MCINNES, I. 2010. Inflammation is present in early human tendinopathy. American Journal of Sports Medicine. 38(10):2085-2091.

https://doi.org/10.1177/0363546510372613

45. NIESEN-VERTOMMEN, S.; TAUNTON, J.; CLEMENT, D.; MOSHER, R. 1992. The Effect of Eccentric Versus Concentric Exercise in the Management of Achilles Tendonitis. Clinical Journal of Sport Medicine. 2:109-113.

https://doi.org/10.1097/00042752199204000-00006

46. NIRSCHL, R.P. 1992. Elbow tendinosis/tennis elbow. Clin Sport Med. 11:851-70.

47. O'NEILL, S.; BARRY, S.; WATSON, P. 2019. Plantarflexor strength and endurance deficits associated with mid-portion Achilles tendinopathy: The role of soleus. Physical Therapy in Sport. 37.69-76. https://doi.org/10.1016/j.ptsp.2019.03.002

48. PAAVOLA, M.; KANNUS, P.; JÄRVINEN, M. 2005. Epidemiology of tendon problems in sport. In: Maffulli, N.; Renström, P.; Leadbetter, W.B. (eds). Tendon Injuries. Springer (London). p.32-39.

https://doi.org/10.1007/1-84628-050-8_5

49. PAAVOLA, M.; KANNUS, P.; PAAKKALA, T.; PASANEN, M.; JÄRVINEN, M. 2000. Longterm prognosis of patients with achilles tendinopathy: An observational 8-year follow-up study. American Journal of Sports Medicine. 28(5):634-642.

https://doi.org/10.1177/036354650002800 50301

50. PARK, Y.H.; KIM, T.J.; CHOI, G.W.; KIM, H. 2019. Achilles tendinosis does not always precede Achilles tendon rupture. Knee Surgery, Sports Traumatology, Arthroscopy. 27(10):32973303.

https://doi.org/10.1007/s00167-018-5172-3

51. PAULUS, J.; CROISIER, J.; KAUX, J.; BURY, T. 2019. Eccentric versus Concentric - Which Is the Most Stressful Cardiovascularly and Metabolically? Current Sports Medicine Reports. 18(12):477-489.

https://doi.org/10.1249/

JSR.0000000000000666
52. PUDDU, G.; IPPOLITO, E.; POSTACHINNI, F. 1976. A classification of Achilles tendon disease. Am J Sport Med. 4:145-50.

53. REINKING, M. 2012. Tendinopathy in athletes. Physical Therapy in Sport. 13(1):3-10. https://doi.org/10.1016/j.ptsp.2011.06.004

54. ROIG, M.; O'BRIEN, K.; KIRK, G.; MURRAY, R.; MCKINNON, P.; SHADGAN, B.; REID, W. 2009. The effects of eccentric versus concentric resistance training on muscle strength and mass in healthy adults: A systematic review with meta-analysis. British Journal of Sports Medicine. 43(8):556-568.

https://doi.org/10.1136/bjsm.2008.051417

55. RUARO, B.; CUTOLO, M.; ALESSANDRI, E.; ZAOTTINI, F.; PICASSO, R.; PISTOIA, F.; FERRARI, G.; MARTINOLI, C. 2019. Don't forget the jumper's knee in the young sportsman: evaluation of patellar tendinopathy with a high frequency ultrasound probe. Reumatismo. 71(3):160-162.

https://doi.org/10.4081/

reumatismo.2019.1231

56. SCHMIKLI, S.L.; BACKX, F.J.G.; KEMLER, H.J.; VAN MECHELEN, W. 2009. National survey on sports injuries in the Netherlands: target populations for sports injury prevention programs. Clinical Journal of Sport Medicine. 19(2):101-106.

https://doi.org/10.1097/

JSM.0b013e31819b9ca3

57. SCOTT, A.; BACKMAN, L.; SPEED, C. 2015. Tendinopathy: Update on Pathophysiology. Journal of Orthopaedic \& Sports Physical Therapy. 45(11):833-841.

https://doi.org/10.2519/jospt.2015.5884

58. SEYNNES, O.; ERSKINE, R.; MAGANARIS, C.; LONGO, S.; SIMONEAU, E.; GROSSET, J.; NARICl, M. 2009. Training-induced changes in structural and mechanical properties of the patellar tendon are related to muscle hypertrophy but not to strength gains. Journal of Applied Physiology. 107(2):523-530.

https://doi.org/10.1152/

japplphysiol.00213.2009

59. STANISH, W.D.; RUBINOVICH, R.; CURWIN, S. 1986. Eccentric exercise in chronic tendinitis. Clin Othop. 208:65-8. 
60. STEFANSSON, S.; BRANDSSON, S.; LANGBERG, H.; ARNASON, A. 2019. Using Pressure Massage for Achilles Tendinopathy: A Single-Blind, Randomized Controlled Trial Comparing a Novel Treatment Versus an Eccentric Exercise Protocol. Orthopaedic Journal of Sports Medicine. 7(3):1-10.

https://doi.org/10.1177/2325967119834284

61. STERGIOULAS, A.; STERGIOULAS, M.; AARSKOG, R.; LOPES-MARTINS, R.; BJORDAL, J. 2008. Effects of low-level laser therapy and eccentric exercises in the treatment of recreational athletes with chronic achilles tendinopathy. American Journal of Sports Medicine. 36(5):881-887.

https://doi.org/10.1177/0363546507312165

62. STEVENS, M.; TAN, C. 2014. Effectiveness of the alfredson protocol compared with a lower repetition-volume protocol for midportion achilles tendinopathy: A randomized controlled trial. Journal of Orthopaedic and Sports Physical Therapy. 44(2):59-67. https://doi.org/10.2519/jospt.2014.4720

63. SUER, M.; ABD-ELSAYED, A. 2019. Tendinopathy. In Abd-Elsayed A. (eds). Pain. Springer (Cham). p.707-710.

https://doi.org/10.1007/978-3-319-991245_151

64. SUSSMILCH-LEITCH, S.; COLLINS, N.; BIALOCERKOWSKI, A.; WARDEN, S.; CROSSLEY, K. 2012. Physical therapies for Achilles tendinopathy: Systematic review and metaanalysis. Journal of Foot and Ankle Research. 5(1). https://doi.org/10.1186/1757-1146-5-15
65. TARPADA, S.; MORRIS, M.; LIAN, J.; RASHIDI, S. 2018. Current advances in the treatment of medial and lateral epicondylitis. Journal of Orthopaedics. 15(1):107-110.

https://doi.org/10.1016/j.jor.2018.01.040

66. TORRECILLAS, J.; SEGURA, M. 2019. Influencia en la participación en actividades de ocio sobre la calidad de vida de las personas con diversidad funcional. In Padilla, D.; Aguilar, J.; López, R. (eds). Salud y Ciclo Vital. 1st ed. Dykinson, S.L. p.51-58.

https://doi.org/10.2307/j.ctvfb6z2h.7

67. VALLEJO, A.; SCHROEDER, E.; ZHENG, L.; JENSKY, N.; SATTLER, F. 2006. Cardiopulmonary responses to eccentric and concentric resistance exercise in older adults. Age and Ageing. 35(3):291-297. https://doi.org/10.1093/ageing/afj082

68. VAN RIJN, D.; VAN DEN AKKER-SCHEEK, I.; STEUNEBRINK, M.; DIERCKS, R.; ZWERVER, J.; VAN DER WORP, H. 2019. Comparison of the Effect of 5 Different Treatment Options for Managing Patellar Tendinopathy: A Secondary Analysis. Clinical Journal of Sport Medicine. 29(3):181-187.

https://doi.org/10.1097/

JSM.0000000000000520 